\title{
Tratamiento quirúrgico de neuromas interdigitales múltiples en un mismo pie. Caso clínico y revisión bibliográfica
}

\section{Surgical treatment of multiple interdigital neuromas in a same foot. Clinical case and literature review}

\author{
José Valero Salas ${ }^{1}$, José Gallart Ortega ${ }^{2}$, David González López ${ }^{3}$ \\ ${ }^{1}$ Diplomado en Podología, Licenciado en Antropología. Especialista en Cirugía Podológica. \\ Doctor por la Universidad de Zaragoza. valeropodologo@yahoo.es \\ ${ }^{2}$ Diplomado en Podología, Licenciado en Antropología. Doctor por la Universidad de Zaragoza. \\ josegallartortega@yahoo.es \\ ${ }_{3}^{3}$ Diplomado en Podología. Máster en Investigación en Cuidados. david.podologo@hotmail.com
}

\author{
Correspondencia: \\ José Valero Salas \\ Centro Podologico José valero Salas \\ c/ Coso, 55 \\ 50001 Zaragoza \\ Correo electrónico: clinicajosevalero@gmail.com
}

Fecha de recepción: 29 de agosto de 2014

Fecha de aceptación: 30 de noviembrel de 2014

Los autores declaran no tener ningún tipo de interés económico o comercial.

\section{RESUMEN}

Se presenta un singular caso de neuromas múltiples, en primer, segundo, tercer y cuarto espacios interdigitales, asociado a hallux abductus valgus/hallux limitus (HAV/HL) y segundo dedo en martillo. Se hace una descripción de los tumores, el procedimiento quirúrgico utilizado para la extirpación quirúrgica de éstos y de las otras patologías del antepié, el tratamiento post-operatorio y el estudio anatomopatológico. Al mismo tiempo se hace una revisión bibliográfica de los diversos estudios hechos acerca de la frecuencia de los neuromas interdigitales múltiples.

Palabras clave: neuromas interdigitales múltiples; podiatría; cirugía.

\begin{abstract}
It is a singular case of multiple neuromas, in first, second, third and fourth interdigital spaces, associated with hallux abductus valgus/hallux limitus (HAV/HL) and second hammertoe. A description of the tumors, the surgical procedure used for the surgical removal of these and other pathologies of the forefoot, post-operative treatment and the histopathological study. At the same time is a literature review of various studies done about the frequency of multiple interdigital neuromas.
\end{abstract}

Keywords: multiple nterdigital neuromas; podiatry, surgery.

Sumario: 1. Introducción. 2. Caso clínico. 3. Material y métodos. 4. Resultados. 5. Discusión. 6. Conclusiones. Agradecimientos. Bibliografía.

Referencia normalizada: Valero Salas, J., Gallart Ortega, J., González López. Tratamiento quirúrgico de neuromas interdigitales múltiples en un mismo pie. Caso clínico y revisión bibliográfica. Rev. Int. Cienc. Podol. 2015; 9(1): 63-73. 


\section{INTRODUCCIÓN}

El neuroma interdigital se define como una alteración degenerativa consecutiva a una reacción fibroblástica perineural e intraneural sobre un nervio digital plantar en la región de su bifurcación hacia los dedos. Esta patología es una de las neuropatías periféricas más comunes en el pie y es generalmente referida como neuroma de Morton, síndrome de Morton o enfermedad de Morton, debido a la publicación de un primer estudio con 12 casos por el cirujano Thomas G. Morton ${ }^{1}$, en 1876. A Lewis Durlacher (1792-1864), cirujano del pie de los reyes ingleses Victoria y Jorge IV, se le atribuye la primera descripción y tratamiento (un apósito plantar), en 1845, de lo que definió como "afección neurálgica del nervio plantar". Sin embargo, antes que la propia descripción clínica, hay documentada una identificación anatómica por parte de Filippo Civinini (1805-1844), médico y anatomista italiano, quien, en 1835 , hace una descripción detallada de "un engrosamiento nervioso en la planta del pie...apenas sobrepasada la diáfisis del III metatarsiano...se engrosa manifiestamente, deja su color blanco/ lácteo para tornarlo rojizo, deviene fusiforme de cilíndrico que era..."3. De un modo claro está describiendo macroscópicamente la patología que diez años más tarde Durlacher definiera como una "afección neurálgica del nervio plantar" y treinta y un años más tarde Thomas Morton hiciese lo propio como "una peculiar y dolorosa afección de la cuarta articulación metatarso-falángica". Sucesivamente, Dana ${ }^{4}$, en 1885, utiliza el término de "luxación podalgia"; Polloson", en 1889, el de "metatarsalgia anterior" y Bradford", en 1891, el de "neuralgia metatarsal". En 1893, TSK Morton ${ }^{7}$ (hijo de TG Morton) denomina por vez primera "metatarsalgia de Morton" a un proceso patológico que ha recibido numerosas denominaciones y que, aún en la actualidad, sigue siendo objeto de controversia en cuanto a su propia denominación, su etiología, histopatología y tratamiento.

El neuroma de Morton, por definición, es un tumor que puede aparecer en cualquier nervio. Como quiera que nos estamos refiriendo a neuromas interdigitales no vamos a incluir entre éstos al neuroma que aparece bajo la cabeza del quinto metatarsiano ${ }^{8}$, pese a que está localizado en la rama más lateral del nervio plantar lateral pero su situación es la zona lateral plantar de la cabeza del quinto metatarsiano $\mathrm{y}$, por tanto, no puede ser incluido en los de localización estrictamente interdigital.

Aunque la incidencia de los neuromas interdigitales en la población general no ha sido determinada, puede ser considerada como una patología muy frecuente. Así lo observó Youngswick en $1994^{9}$ ya que un $9,3 \%$ de 4.000 pacientes que se presentaron en su consulta con complicaciones en el pie fueron diagnosticados de neuroma de Morton.

Aunque se han propuesto otras muchas causas (alteraciones o variantes anatómicas en los metatarsianos o en los nervios periféricos distales o el uso de calzado antifisiológico $\left.{ }^{10,11,12,13}\right)$, está generalmente aceptado que, originariamente, la etiología más frecuente de los neuromas interdigitales del pie es traumática o microtraumática/ patomecánica. Entre las causas patomecánicas caben destacarse los patrones biomecánicos pronadores como condición a menudo observada en los pies con neuroma de Morton ${ }^{14}$. Autores como Gianni et al. ${ }^{15}$, consideran que la hiperpronación o pronación patológica produce inestabilidad del antepié en la fase de propulsión y causa excesiva tracción y tensión de los nervios digitales plantares. El antepié varo o supinado (el cual se asocia a menudo con una pronación subastragalina excesiva o a una insuficiencia de primer radio) es considerado otro factor influyente, Bartolomei y Wertheimer, en $1983^{16}$, además de hallar que el $85 \%$ de los pacientes con neuromaa tenían sobrepeso, describieron que tres cuartas partes de los neuromas unilaterales se producen en pacientes con antepié varo y la mayor parte de los neuromas bilaterales se presentan con un antepié valgo flexible. Los pies cavos $^{17}$ y el pie equino ${ }^{18}$ también han sido considerados factores causales debido al incremento de tensión de la fascia plantar y del ligamento intermetarsiano transverso.

Los neuromas múltiples han sido considerados como excepcionales por numerosos autores $^{19,20}$ aunque, en la actualidad, diversos estudios demuestran que pueden ser considerados incluso más frecuentes que los aislados. Por otra parte, la asociación neuroma/HAV ha sido demostrada por diversos autores ${ }^{21,22}$. Otros autores, incluso, consideran que el neuroma es una consecuencia del $\mathrm{HAV}^{23}$. 
Se presenta un caso clínico excepcional en el que se asociaban el HAV, el segundo dedo en martillo y neuromas en los cuatro espacios interdigitales del pie derecho. Al mismo tiempo se hace una revisión bibliográfica acerca de la frecuencia de los neuromas interdigitales múltiples en los pies.

\section{CASO CLÍNICO}

Paciente de 72 años de edad que refiere intenso dolor en todo el antepié, especialmente en la zona distal de las articulaciones metatarsofalángicas de su pie derecho. El dolor se agrava con la deambulación, aunque se manifiesta, con similar intensidad, incluso en reposo. La exploración física, que resulta difícil por el intenso dolor que produce la más leve manipulación. Muestra una marcada limitación de la primera articulación metatarso-falángica con HAV, segundo dedo en martillo y discreta inflamación de partes blandas en las articulaciones metatarso-falángicas $2^{\mathrm{a}}, 3^{\mathrm{a}}$ y $4^{\mathrm{a}}$ (Figura 1 ).

El examen radiológico muestra HAV con evidentes signos degenerativos en la primera articulación metatarso-falángica de su pie derecho y segundo dedo en martillo y supraducción (Figura 2).

Se solicita estudio ecográfico que revela una formación nodular hipoecoica de unos $8 \mathrm{~mm}$. a nivel del primer espacio intermetatarsiano, compatible con neuroma-bursitis, y sendas formaciones nodulares hipoecoicas a nivel de los espacios intermetatarsianos $2^{\circ}, 3^{\circ}$ y $4^{\circ}$, de 19 , 13 y $10 \mathrm{~mm}$. respectivamente, compatibles con neuromas de Morton (Figura $3 \mathrm{a}, \mathrm{b}, \mathrm{c}, \mathrm{d}$ ).

$\mathrm{Su}$ historia médica revela que padece diabetes mellitus tipo I (insulino-dependiente), hipertensión arterial y alteraciones vasculares periféricas, todo ello bajo control y tratamiento medicamentoso y dietético. Ninguna de estas patologías supone una contraindicación absoluta para la realización de cirugía bajo anestesia local y régimen ambulatorio.

\section{Procedimiento quirúrgico}

Se procede, en primer lugar a la artroplastia tipo Keller con una incisión dorsal, paralela al extensor propio del hallux, a través de la cual se extirpa el neuroma en el primer espacio inter-

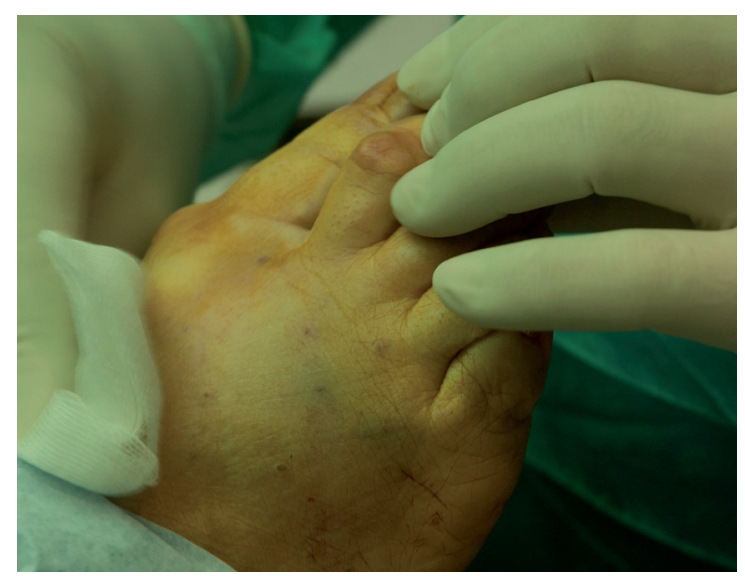

Figura 1. Aspecto pre-operatorio.

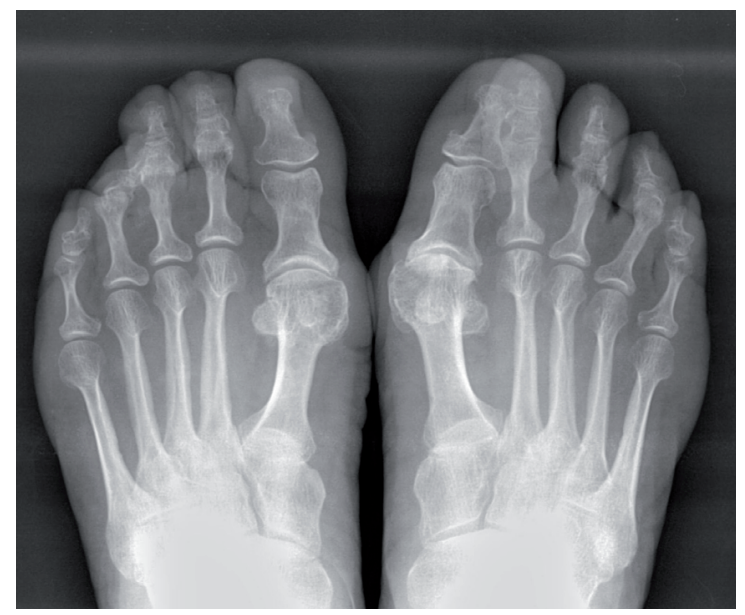

Figura 2. Radiografía pre-operatoria.

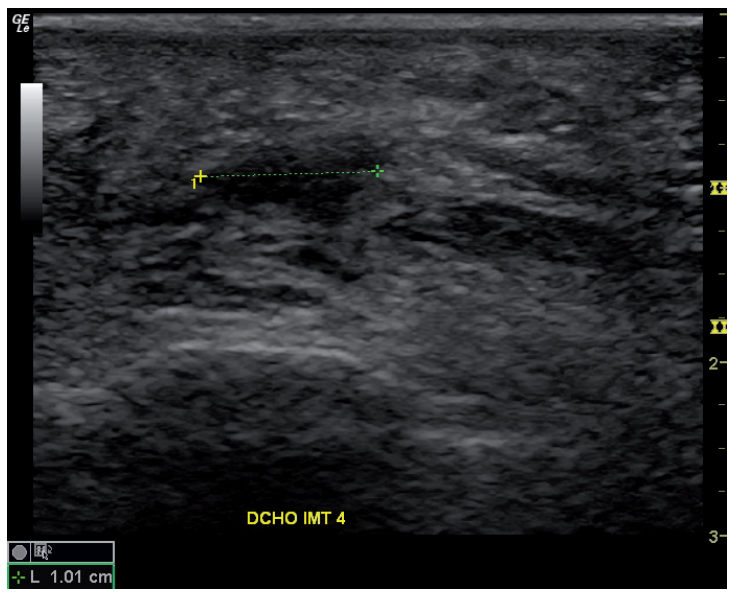

Figura 3a. Ecografía del primer espacio intermetatarsiano que muestra imagen compatible con neuroma/bursitis. 


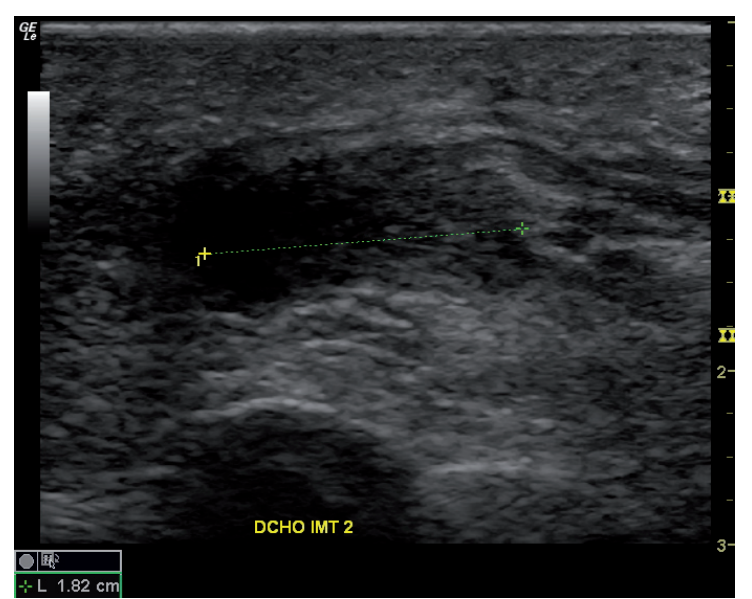

Figura 3b. Ecografía del segundo espacio intermetatarsiano.

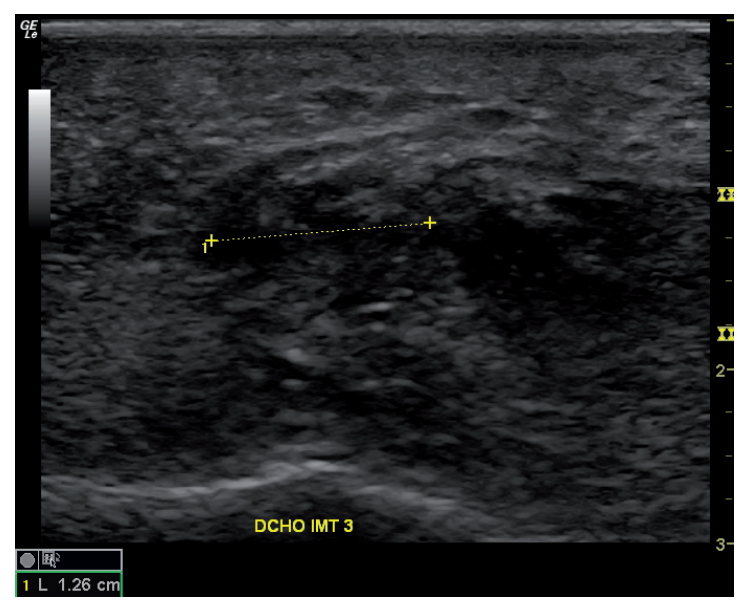

Figura 3c. Ecografía del tercer espacio intermetatarsiano.

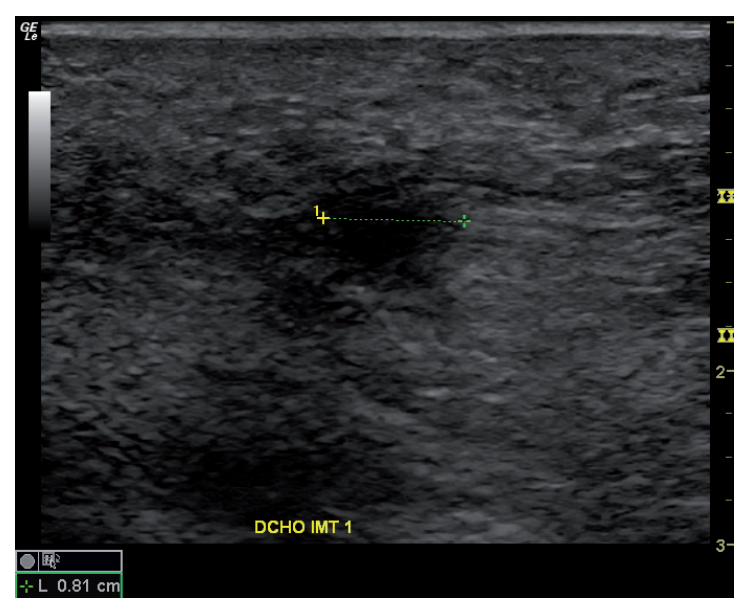

Figura 3d. Ecografía del cuarto espacio intermetatarsiano. digital (Figura 4a). Se realiza una segunda incisión en el segundo espacio inter metatarso-digital, desde el cuello del segundo metatarsiano hasta la zona media del espacio interdigital, a través de la que se extirpa el neuroma en segundo espacio (Figura 4b). Otras dos incisiones en tercero y cuarto espacios, con el mismo diseño que la utilizada en el segundo, se practican para extirpar los neuromas en tercero y cuarto espacios (Figuras 4c y 4d). Se sutura por planos: en planos internos con sutura absorbible de $3 / 0 \mathrm{y}$ $4 / 0$ y la piel con monofilamento de polipropileno de 4/0 (Figura 4e).

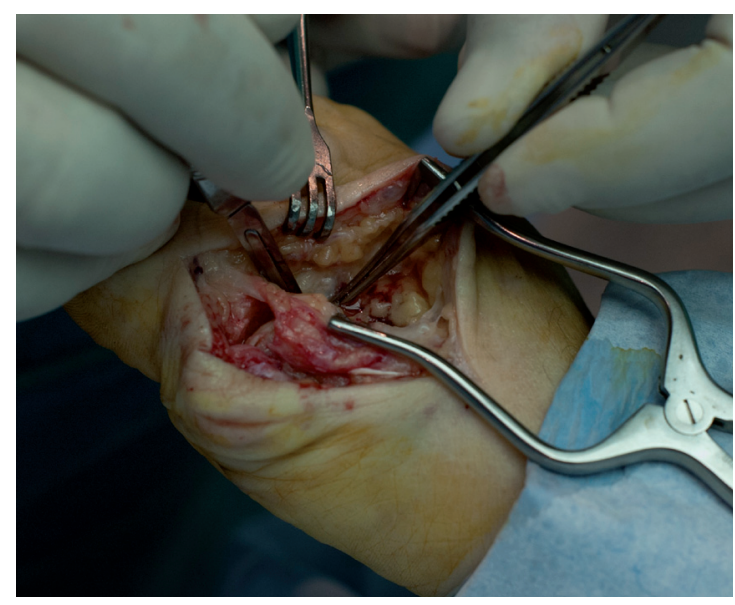

Figura 4a. Resección del neuroma en el primer espacio.

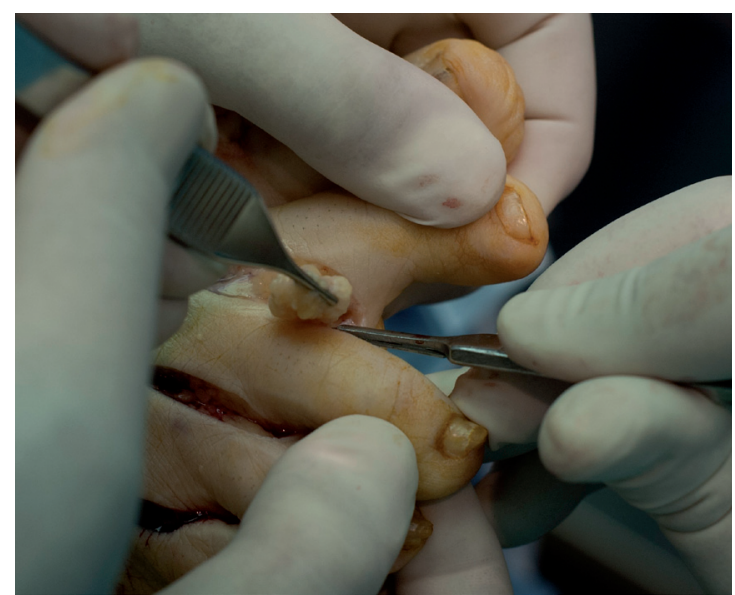

Figura 4b. Resección del neuroma en el segundo espacio. 


\section{Tratamiento post-quirúrgico}

La pieza extraída se remitió para su estudio anatomopatológico. Se prescribió antibioterapia por vía oral (500 mg de amoxicilina cada 8 horas) durante quince días. Se indicó reposo absoluto durante las primeras veinticuatro horas y relativo, con calzado cómodo, durante las tres semanas siguientes. La retirada de la sutura tuvo lugar a los 14 días de la intervención.

Previo estudio funcional, el tratamiento ortopodológico consistió en soportes plantares confeccionados con polipropileno de $2 \mathrm{~mm}$., neutralizando la articulación subastragalina, con elementos estabilizadores anteriores confeccionados con material blando.

Un año después de la intervención la paciente presenta un excelente aspecto estético y funcional, han desaparecido por completo los dolores y se muestra absolutamente satisfecha (Figura 5).

\section{Estudio anatomopatológico}

Descripción macroscópica: Cuatro fragmentos que miden $2,3 \times 0,8 \times 0,5 \mathrm{~cm}$ (primer espacio), $2 \times 1,5 \times 0,5 \mathrm{~cm}$ (segundo espacio), 1,8x1,2x0,5 $\mathrm{cm}$ (tercer espacio) y $1,7 \times 0,8 \times 0,5 \mathrm{~cm}$ (cuarto

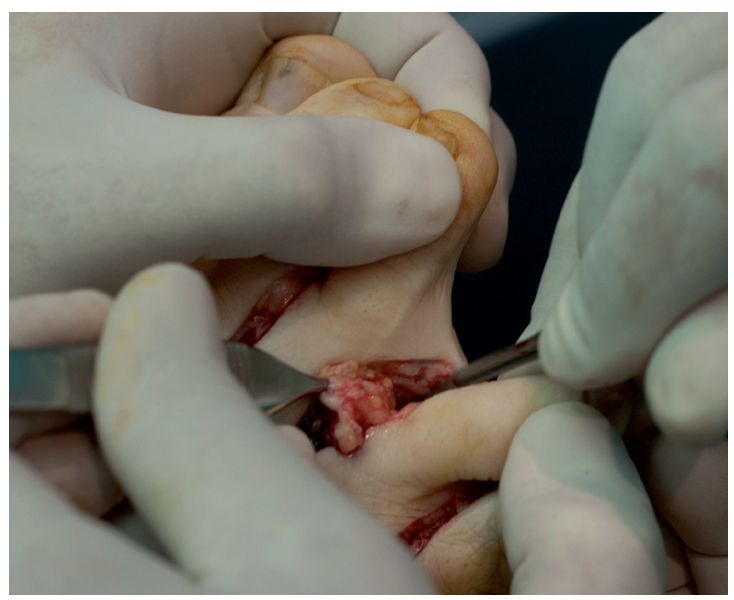

Figura 4c. Resección del neuroma en el tercer espacio.

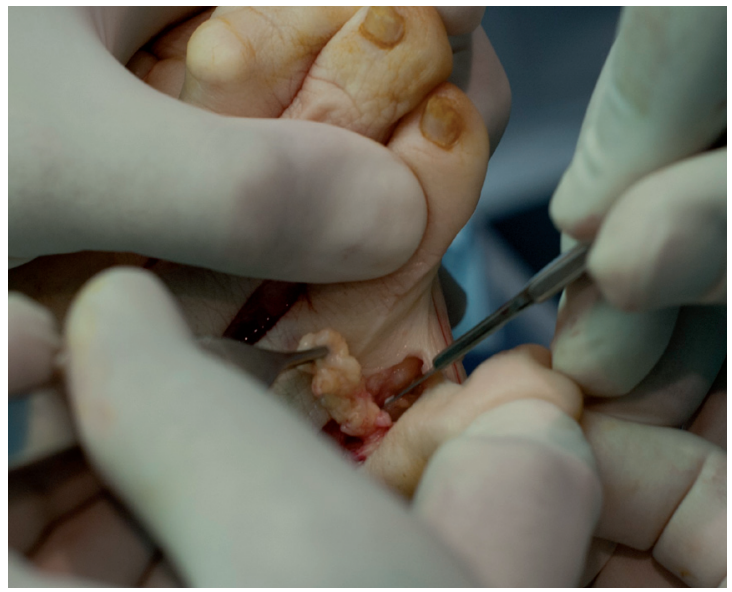

Figura 4d. Resección del neuroma en el cuarto espacio.

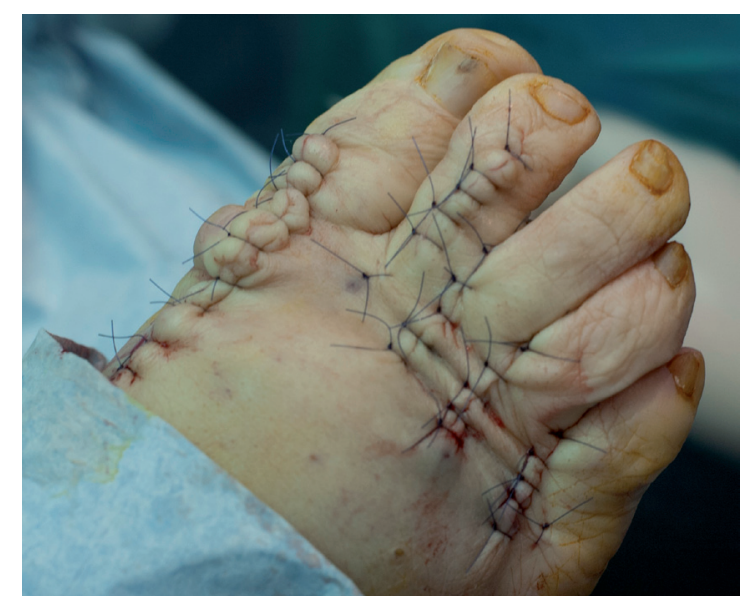

Figura 4e. Suturas. Aspecto final.

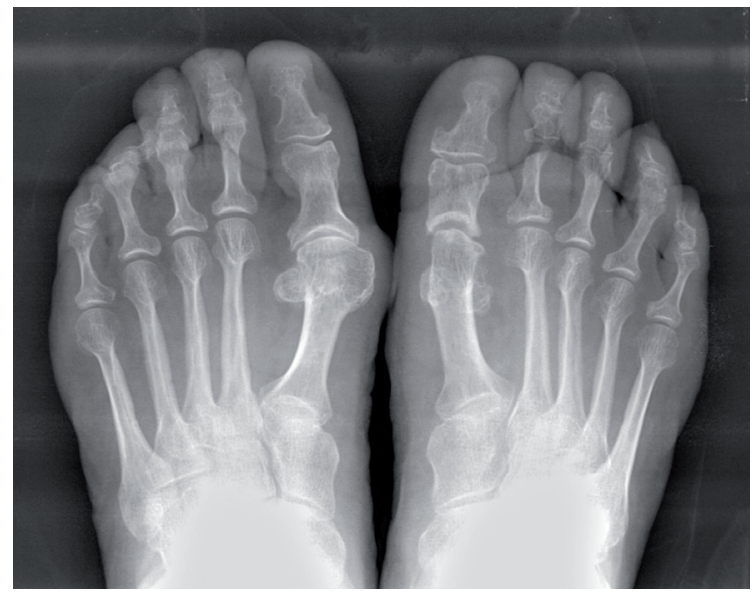

Figura 5a. Radiografía post-operatoria: un año después de la intervención. 


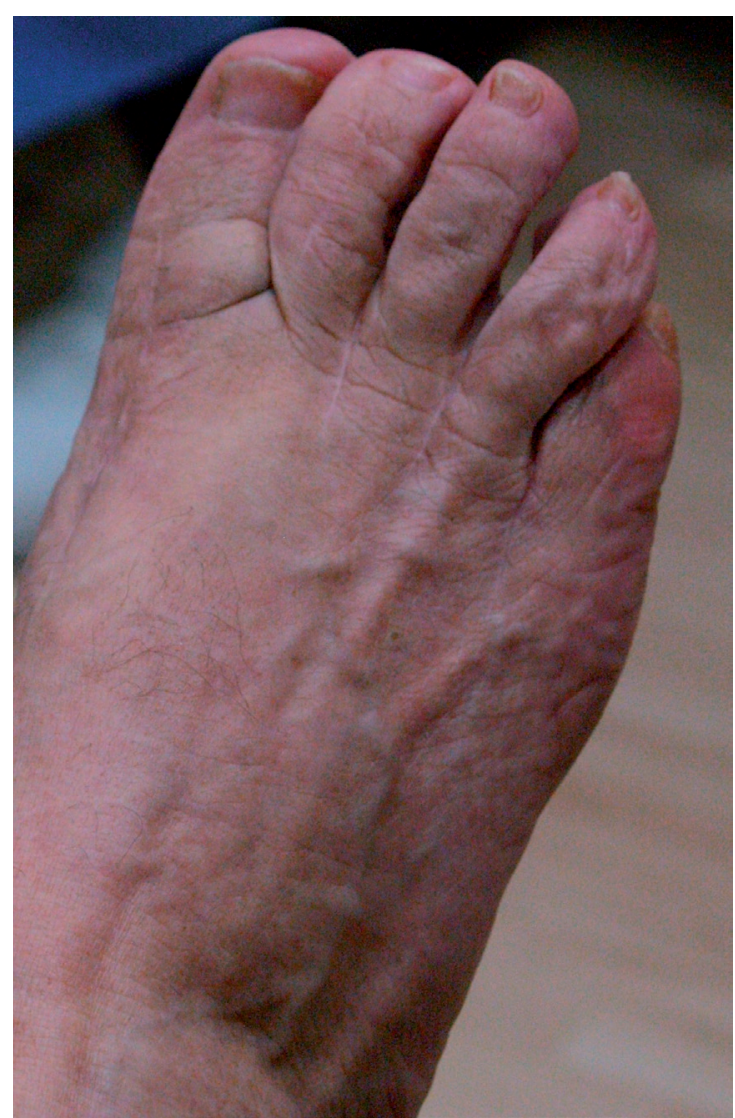

Figura 5b. Aspecto del antepié un año después de la intervención. espacio) con aspecto fibroadiposo (Figura 6). Se incluyen varios fragmentos representativos.

Descripción microscópica: Las secciones estudiadas están constituidas por tejido fibro-adiposo en los que se reconoce una proliferación de fibras nerviosas con fibrosis peri e intraneural así como algunos vasos arteriolares con una disminución de su lumen.

Diagnóstico anatomopatológico: Compatible con neuromas de Morton de $1^{\circ}, 2^{\circ}, 3^{\circ}$ y $4^{\circ}$ espacios intermetatarsianos del pie derecho.

\section{MATERIAL Y MÉTODOS}

Con la finalidad de hacer una revisión acerca de la literatura acerca de los neuromas múltiples, se hizo una búsqueda en Medline, PubMed y Google Académico utilizando las siguientes palabras clave: "neuroma de Morton", "neuroma interdigital", "enfermedad de Morton" y "fibrosis perineural de Morton". Como límites se utilizaron las palabras "pie", "múltiples" y "tratamiento quirúrgico". Todas ellas en tres idiomas (español, inglés y francés). En una primera búsqueda no se utilizó ningún tipo de límite temporal, aunque posteriormente, a fin de hacer una selección más actual se utilizó el límite "1980-2014".

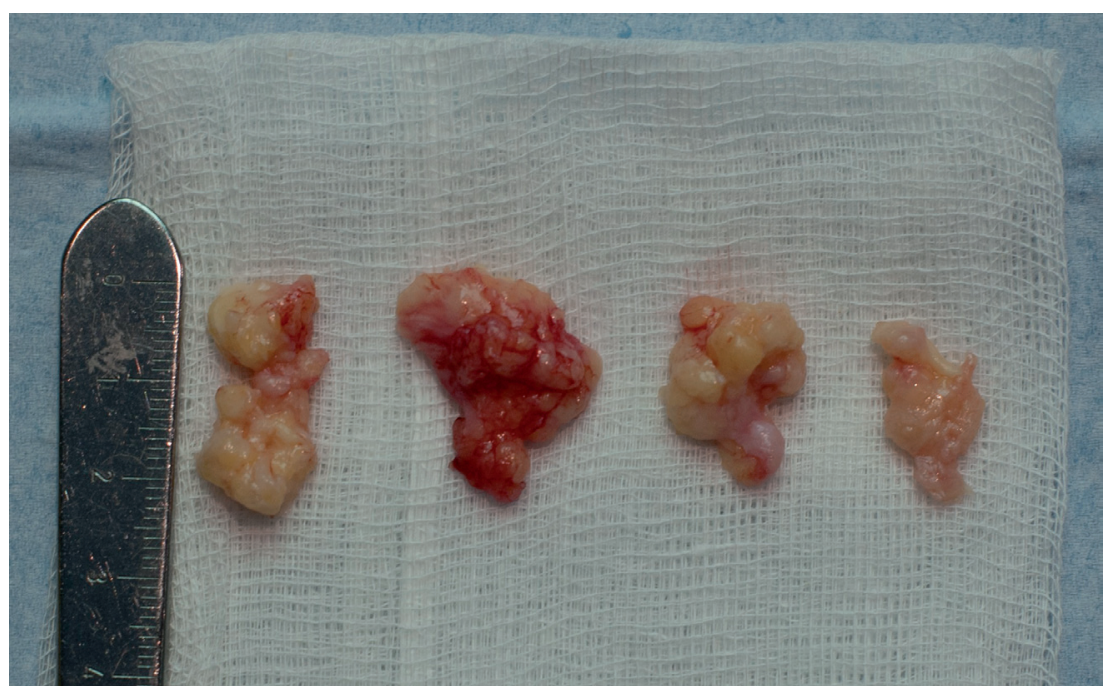

Figura 6. Piezas extirpadas. 


\section{RESULTADOS}

En una primera búsqueda se obtuvieron 407 referencias de artículos que se referían a los neuromas interdigitales (con diversas denominaciones), aunque en la mayoría de ellos no se trataba de la condición de multiplicidad y otros se referían a aspectos genéricos que no trataban estudios completos de casos intervenidos quirúrgicamente. Una nueva búsqueda, con el límite "múltiples", redujo muy sensiblemente el número de referencias a consultar a 43. Sin embargo, en muchos artículos no se especificaba la localización y en otros no quedaba claro si era en un sólo pie o en los dos. Se han excluido aquellos artículos en los que el diagnóstico de neuroma no estaba ratificado por estudio histopatológico posterior. Por tanto, se han seleccionado los estudios más completos y en los que, como mínimo se cumplían una serie de condiciones: número total de neuromas extirpados con diagnóstico ratificado histopatológicamente, porcentaje (o cantidad) en cada uno de los espacios y condición de singularidad o pluralidad en un mismo pie. De este modo, se obtuvieron 12 estudios con todas las características requeridas para su inclusión, 8 en inglés, 1 en francés y 3 en español. Los resultados fueron los siguientes:

Gudas y Mattana ${ }^{24}$, en un estudio de 1986, con 43 pacientes ( 36 mujeres y 7 hombres) a los que extirparon un total de 59 neuromas, hallaron $51(86,4 \%)$ en el tercer espacio, $5(8,5 \%)$ en el cuarto espacio y solo $3(5,1 \%)$ en el segundo. No hallaron neuromas en el primer espacio ni neuromas múltiples. Addante et al. ${ }^{25}$ en un estudio realizado en el mismo año, sobre 143 pies de 136 pacientes a los que extirpó quirúrgicamente 152 neuromas, obtuvo los siguientes resultados: 109 (80\%) pacientes eran mujeres y $27(20 \%)$ eran hombres. El rango de edad de los pacientes iba de los 22 a los 67 años. 8 pacientes $(5,9 \%)$ fueron intervenidos de neuromas bilaterales. El espacio más frecuentemente afectado fue el tercero con 101 casos $(66,4 \%)$; el segundo espacio estaba afectado en 27 casos $(17,8 \%)$, seguido por el primer espacio con 6 casos $(3,9 \%)$. Por último, el cuarto espacio estaba afectado en 4 casos (2,6\%). 14 (9,2\%) neuromas fueron extirpadas en otras áreas del pie, 7 pacientes $(5,1 \%)$ presentaron neuromas en dos espacios y 1 paciente en tres espacios interdigitales del mismo pie.

Arriaza et al. ${ }^{19}$, en 2000, hace una revisión de 44 neuromas interdigitales intervenidos en 24 pacientes hallando que 24 de ellos aparecieron en el segundo espacio y 20 neuromas en el tercer espacio. En 6 pacientes extirpan neuromas en dos espacios del mismo pie y en tres de estos pacientes los neuromas múltiples aparecieron en los dos pies.

Esling et al. ${ }^{26}$, en 2002, en un estudio sobre 39 neuromas extirpados, hallan que 27 pies $(82 \%)$ tenían un único neuroma y $6(8 \%)$ tenían más de uno. En el caso de los neuromas únicos, el segundo espacio estaba afectado en 3 pies $(9 \%)$ y el tercer espacio en 23 pies $(70 \%)$ y el primer espacio en un caso. En el caso de los múltiples, el segundo y el tercer espacios estaban afectados por igual. Fernández et al. ${ }^{27}$, también en 2002, sobre un total de 24 pacientes y 32 pies afectados de neuroma, sólo halló neuromas en el segundo $(28,1 \%)$ y en el tercer espacios $(71,9 \%)$ mientras que 6 pacientes $(25 \%)$ tuvieron afectación bilateral.

Hewitt et al. ${ }^{28}$, en 2007, hacen un estudio de 60 casos, encontrando que en el tercer espacio hay un $86,3 \%$ de los neuromas, en el segundo espacio un $13,7 \%$, y neuromas múltiples en el mismo pie el $13,7 \%$ de los casos. Por su parte Nery et al. ${ }^{29}$, en el mismo año pero analizando 264 casos, hallan un 83,5\% de neuromas en el tercer espacio, en el segundo espacio aparecen un $15 \%$, en el primer espacio un $1,5 \%$ y neuromas múltiples en ambos pies un 7,5\% de los casos. En 2009, Lee et al. ${ }^{30}$, analizan 210 casos, obteniendo los siguientes resultados: un $46 \%$ de neuromas en el tercer espacio, un $43 \%$ en el segundo espacio y neuromas múltiples en un mismo pie en el 66\% de los casos. En 2010, Soo et al. ${ }^{31}$, estudian 60 casos, hallando un $43 \%$ de los neuromas en el tercer espacio, un $57 \%$ en el segundo y neuromas múltiples en el 13\% de los casos. Faraj y Hosur ${ }^{32}$, en 1010, con 42 casos, refieren 17 casos $(40 \%)$ en el segundo espacio y $25(60 \%)$ en el tercero. Por otra parte, no hallan neuromas ni en el primero ni en el cuarto espacios interdigitales ni presentaciones múltiples.

González et al. ${ }^{21}$ presentan una muestra constituida por 76 personas, 72 mujeres $(94,7 \%)$ y 4 hombres $(5,3 \%)$, que fueron intervenidas quirúrgicamente de neuromas interdigitales. De 
éstas, $51(67,1 \%)$ fueron intervenidas de neuromas en un sólo pie y $25(32,9 \%)$ fueron intervenidas en ambos pies, resultando un total de 101 pies intervenidos. En el caso de las mujeres, $24(31,6 \%)$ fueron intervenidas de neuromas en ambos pies, mientras que $48(68,4 \%)$ fueron intervenidas de un sólo pie. En el caso de los hombres sólo en un caso $(1,3 \%)$ se intervino de neuromas en ambos pies. El seguimiento post-operatorio medio de los pacientes fue de 17,93 meses. La edad media de los pacientes intervenidos es de 57,75 años, siendo de 57,97 para las mujeres y 53,75 para los hombres. Respecto al diagnóstico por imagen, fue positivo en todas las personas de la muestra. En 71 casos $(93,4 \%)$ el diagnóstico se realizó por medio de ecografía y en $5(6,6 \%)$ por resonancia magnética nuclear. Atendiendo al espacio interdigital donde se localizaban los neuromas, de los 101 pies solo $10(9,9 \%)$ presentaron un único espacio con neuroma, de los cuales en 6 casos se localizó en el tercer espacio y 4 en el segun- do. Los 91 pies restantes $(90,1 \%)$ presentaron más de un espacio interdigital con neuroma: 88 pies $(87,12 \%)$ presentaba neuroma en segundo y tercer espacio interdigitales conjuntamente, 1 presentaba neuroma en segundo, tercer y cuarto espacio y 2 pies con neuroma en tercer y cuarto espacios interdigitales.

En un reciente estudio de Valero et al. ${ }^{33}$, en el que el diagnóstico de neuroma está confirmado por estudios ecográfico y anatomopatológico, de los 279 pies intervenidos, 262 corresponden a mujeres $(93,9 \%)$ y 17 casos a hombres $(6,03 \%) .97$ pies $(34,8 \%)$ presentaron un sólo neuroma, de los cuales $72(74,2 \%$, ) se localizaron en el tercer espacio, $24(24,7 \%)$ en el segundo espacio y solo $1(1,1 \%)$ en el cuarto espacio. 182 pies $(65,2 \%)$ presentaron neuromas múltiples: 179 en el segundo y tercer espacios, 2 pacientes en el tercero y en el cuarto espacios y un paciente presentó tres neuromas que afectaban al segundo, tercer y cuarto espacios (Tabla 1).

\begin{tabular}{|c|c|c|c|c|c|c|}
\hline Autor & $\mathbf{N}^{0}$ neuromas & $1^{\circ}$ espacio & $2^{\circ}$ espacio & $3^{\circ}$ espacio & $4^{\circ}$ espacio & Multiples \\
\hline $\begin{array}{l}\text { Gudas y } \\
\text { Mattana (1986) }\end{array}$ & 59 & $\begin{array}{r}0 \\
0 \%\end{array}$ & $\begin{array}{r}3 \\
5,1 \%\end{array}$ & $\begin{array}{r}51 \\
86,4 \%\end{array}$ & $\begin{array}{r}5 \\
8,5 \%\end{array}$ & $\begin{array}{r}0 \\
0 \%\end{array}$ \\
\hline $\begin{array}{l}\text { Addante et al. } \\
\text { (1986) }\end{array}$ & 152 & $\begin{array}{r}6 \\
3,9 \%\end{array}$ & $\begin{array}{r}27 \\
17,8 \%\end{array}$ & $\begin{array}{r}101 \\
66,4 \%\end{array}$ & $\begin{array}{r}4 \\
2,6 \%\end{array}$ & $\begin{array}{r}7 \\
5.1 \%\end{array}$ \\
\hline $\begin{array}{l}\text { Arriaza et al. } \\
(2000)\end{array}$ & 44 & $\begin{array}{r}0 \\
0 \%\end{array}$ & $\begin{array}{r}24 \\
54,5 \%\end{array}$ & $\begin{array}{r}20 \\
45,5 \%\end{array}$ & $\begin{array}{r}0 \\
0 \%\end{array}$ & $\begin{array}{r}18 \\
40,9 \%\end{array}$ \\
\hline $\begin{array}{l}\text { Esling et al. } \\
(2002)\end{array}$ & 39 & $\begin{array}{r}1 \\
2,5 \%\end{array}$ & $\begin{array}{r}9 \\
23 \%\end{array}$ & $\begin{array}{r}29 \\
74,3 \%\end{array}$ & $\begin{array}{r}0 \\
0 \%\end{array}$ & $\begin{array}{r}6 \\
18 \%\end{array}$ \\
\hline $\begin{array}{l}\text { Fernández et } \\
\text { al. (2002) }\end{array}$ & 32 & $\begin{array}{r}0 \\
0 \%\end{array}$ & $\begin{array}{r}9 \\
28.1 \%\end{array}$ & $\begin{array}{r}23 \\
71.9 \%\end{array}$ & $\begin{array}{r}0 \\
0 \%\end{array}$ & $\begin{array}{r}0 \\
0 \%\end{array}$ \\
\hline $\begin{array}{l}\text { Hewitt et al. } \\
(2007)\end{array}$ & 60 & $\begin{array}{r}0 \\
0 \%\end{array}$ & $\begin{array}{r}8 \\
13.7 \%\end{array}$ & $\begin{array}{r}52 \\
86.3 \%\end{array}$ & $\begin{array}{r}0 \\
0 \%\end{array}$ & $\begin{array}{r}8 \\
13.7 \%\end{array}$ \\
\hline $\begin{array}{l}\text { Nery et al. } \\
(2007)\end{array}$ & 264 & $\begin{array}{r}4 \\
1.5 \%\end{array}$ & $\begin{array}{r}40 \\
15 \%\end{array}$ & $\begin{array}{r}220 \\
83.5 \%\end{array}$ & $\begin{array}{r}0 \\
0 \%\end{array}$ & $\begin{array}{r}20 \\
7.5 \%\end{array}$ \\
\hline $\begin{array}{l}\text { Lee et al. } \\
(2009)\end{array}$ & 210 & $\begin{array}{r}17 \\
8.1 \%\end{array}$ & $\begin{array}{r}98 \\
46.7 \%\end{array}$ & $\begin{array}{r}92 \\
43.8 \%\end{array}$ & $\begin{array}{r}3 \\
1.4 \%\end{array}$ & $\begin{array}{r}138 \\
66 \%\end{array}$ \\
\hline $\begin{array}{l}\text { Soo et al. } \\
(2010)\end{array}$ & 60 & $\begin{array}{r}0 \\
0 \%\end{array}$ & $\begin{array}{r}34 \\
57 \%\end{array}$ & $\begin{array}{r}26 \\
43 \%\end{array}$ & $\begin{array}{r}0 \\
0 \%\end{array}$ & $\begin{array}{r}8 \\
13 \%\end{array}$ \\
\hline $\begin{array}{l}\text { Faraj y Hosur } \\
(2010)\end{array}$ & 42 & $\begin{array}{r}0 \\
0 \%\end{array}$ & $\begin{array}{r}17 \\
40 \%\end{array}$ & $\begin{array}{r}25 \\
60 \%\end{array}$ & $\begin{array}{r}0 \\
0 \%\end{array}$ & $\begin{array}{r}0 \\
0 \%\end{array}$ \\
\hline $\begin{array}{l}\text { González et al. } \\
\text { (2013) }\end{array}$ & 189 & $\begin{array}{r}0 \\
0 \%\end{array}$ & $\begin{array}{r}92 \\
48.7 \%\end{array}$ & $\begin{array}{r}94 \\
49.7 \%\end{array}$ & $\begin{array}{r}3 \\
1.6 \%\end{array}$ & $\begin{array}{r}170 \\
90 \%\end{array}$ \\
\hline $\begin{array}{l}\text { Valero et al. } \\
\text { (2014) }\end{array}$ & 462 & $\begin{array}{r}0 \\
0 \%\end{array}$ & $\begin{array}{r}204 \\
44,15 \%\end{array}$ & $\begin{array}{r}254 \\
54,97 \%\end{array}$ & $\begin{array}{r}4 \\
0,86 \%\end{array}$ & $\begin{array}{r}362 \\
65.2 \%\end{array}$ \\
\hline
\end{tabular}

Tabla 1. Frecuencia y porcentaje de los neuromas interdigitales en función de los espacios afectados, según diferentes autores. 


\section{DISCUSIÓN}

Desde la simple denominación, la descripción y la etiopatogenia hasta las diversas formas de tratamiento, el neuroma de Morton sigue siendo objeto de controversia.

La localización de los neuromas interdigitales, según diversos autores, es diversa aunque, tradicionalmente, se consideraba que el tercer espacio intermetatarsal es el más frecuentemente afectado, seguido por el segundo espacio intermetatarsal, siendo bastante rara la afectación en el primer y cuarto espacios. Las lesiones pueden ocurrir también bilateralmente y puede encontrarse más de un espacio afectado en el mismo pie, siendo la frecuencia de esta condición muy distinta según los autores. Sin embargo, a la vista de los resultados obtenidos (Tabla 1), especialmente en los estudios de la última década, parece ser que la frecuencia de los neuromas en el segundo y tercer espacios interdigitales es muy similar y que comienza a ser habitual la presencia de neuromas múltiples, algo que se puede constatar en los estudios con un número elevado de casos ${ }^{21,30,33}$, no así cuando los casos no llegan al centenar ${ }^{19,24-28,31}$. Sin embargo la presencia de neuromas en el primero y en el cuatro espacios, sea cual sea el número de casos estudiados, al menos en lo que concierne a ser susceptibles de intervención quirúrgica, sigue siendo casi anecdótica. Por este motivo el caso que se ha presentado en este artículo es especialmente singular.

Este tipo de lesión es más común en las mujeres que en los hombres, siendo su incidencia en las mujeres entre 4 y 18 veces mayor ${ }^{30,34,35}$, Por nuestra parte, en un estudio reciente ${ }^{33}$, coincidiendo con numerosos autores citados en este artículo, hemos hallado que la incidencia de los neuromas interdigitales en las mujeres de 16 veces mayor que en los hombres. Ello puede ser debido a la compresión interdigital y metatarso-digital provocada por el calzado de tacón excesivo y de puntera estrecha. Aunque puede presentarse a cualquier edad, está generalmente aceptado que es más frecuente entre los 40 y los 60 años ${ }^{19,21,32,33}$.

El tratamiento quirúrgico de los neuromas interdigitales también sigue siendo objeto de controversia. Entre los autores que proponen la neurectomía convencional, hay opiniones encontradas en cuanto al abordaje más apropiado y se sigue discutiendo si el mejor es el dorsal o el plantar ${ }^{32,36,37}$. Por otra parte, desde hace bastantes años y en la actualidad, hay dos tendencias predominantes en el tratamiento quirúrgico de los neuromas interdigitales del pie: quienes proponen la neurectomía ${ }^{21,24,25,38,39,40} \mathrm{y}$ quienes están a favor de la neurolisis ${ }^{41,42,43}$ o la termoneurolisis ${ }^{44,45}$. También hay autores que, desde hace muchas décadas ${ }^{46}$ y en la actuali$\mathrm{dad}^{47}$, siguen proponiendo osteotomías metatarsales como tratamiento quirúrgico, sustitutivo de la ablación quirúrgica, de los neuromas interdigitales. Aunque somos partidarios de la neurectomía y consiguiente estudio anatomopatológico de las piezas extirpadas, sin entrar en discutir cualquiera de todas estas opciones quirúrgicas, coincidimos con la mayoría de los autores en que la cirugía es la mejor alternativa sólo cuando han fracasado los tratamientos conservadores $^{48}$. Por otra parte, coincidiendo con otros autores ${ }^{49}$, consideramos que el tratamiento quirúrgico no sustituye o excluye el tratamiento compensador de la alteración patomecánica que desencadenó los neuromas interdigitales.

\section{CONCLUSIONES}

1. La presencia de neuromas en varios espacios intermetatarsales de un mismo pie debe ser considerada como posible o incluso como frecuente en los espacios interdigitales segundo y tercero.

2. Aunque la presencia de los neuromas interdigitales en primero y cuarto espacios puede ser considerada como infrecuente, se sugiere que la exploración física y por técnicas complementarias (ecografía y/o resonancia magnética) incluya todos los espacios intermetatarsales.

\section{AGRADECIMIENTOS}

A la Dra. Reyes Yñigo, MD, por su valiosa colaboración en el estudio ecográfico del caso presentado. 


\section{BIBLIOGRAFÍA}

1. Morton TG. A peculiar and painful affection of the fourth metatarso-phalangeal articulation. Am J Med Sci. 1876;71:37-45.

2. Durlacher L. A Treatise on Corns, Bunions, the Diseases of Nail and the General Management of the Feet. London Simkin, Marshall and Co, 1845, p.52.

3. Civinini F. Su d'un nervoso gangliare rigonfiamento alla pianta del piede. Lettera anatomica al Dr.Salomone Lampronti. Pistoia, Tip. Bracali, 1835.

4. Dana CL. The acro-neuroses. Functional nervous affections of the extremities. Med Rec 1885; $28: 85$.

5. Polloson A. De la metatarsalgie anterieure. La Province Med 1889;6:1.

6. Bradford MA. Metatarsal neuralgia. Boston Med Surg J 1891;125:52.

7. Morton TSK. Metatarsalgia (Morton's painful affection of the foot), with an account of six cases cured by operation. Ann Surg 1893;17:680.

8. Valero J, Gallart J, González D, Agustín L, Marquina R, Deus J, Lahoz M. Neuroma under the fifth metatarsal head. A retrospective study. Histol Histopathol. 2012;27: 1103-7.

9. Youngswwick FD. Intermetatarsal neuroma. Clin Podiatr Med Surg. 1994;11:579-592.

10. Amis JA, Siverhus SW, Liwnicz BH. An anatomic basis for recurrence after Morton's neuroma excision. Foot Ankle. 1992;13(3):153-6.

11. Cantos B, Arriaza R, Del Cerro M. Diagnóstico ecográfico del neuroma de Morton. Rev Ortop Traumatol. 1993;37:468-70.

12. Wu KK. Morton's interdigital neuroma: a clinical review of its etiology, treatment and results. J Foot Ankle Surg. 1996;35(2):112-9; discussion 187-8.

13. Reinherz RP, Weils LS. Morton's interdigital neuroma: a clinical review of its etiology, treatment and results (letter). Foot Ankle Surg. 1996;35(2):187-8.

14. Valero J, Gallart J, González D, Marquina R, Agustín L. Tumores de origen patomecánico. Podología Clínica, 2012;13(1):11-27.

15. Giannini S, Bacchini P, Ceccarelli F, Vannini F. Interdigital neuroma: clinical examination and histhopatological results in 63 cases treated with excision. Foot Ankle Int 2004; 25(2): 79-84.

16. Bartolomei FJ, Wertheimer SJ. Intermetatarsal neuromas: distribution and etiologic factors. J Foot Surg 1983;22:279-82.

17. Wachter SD, Nilson RZ, Thul JR. The relationship between foot structure and intermetatarsal neuromas. J Foot Surg. 1984; 23:436-43.

18. Barrett SL, Jarvis J. Equinus deformity as a factor in forefoot nerve entrapment: treatment with endoscopic gastrocnemius recession. J Am.Podiatr Med Ass. 2005;95(5):464-68.

19. Arriaza R, Baamonde C, Rodríguez C, Cantos B, Sampedro C. Resultados del tratamiento quirúrgico de los neuromas de Morton. Rev Ortop Traumatol 2000;1:35-40.

20. Thomson FN, Deland JT. Occurrence of two interdigital neuromas in the one foot. Foot Ankle 1993;14(1):15-7.

21. González D, Valero J, Gallart J. Estudio de 76 pacientes intervenidos quirúrgicamente de neuromas interdigitales y su relación con la patología del primer radio. Podología Clínica 2013;14(4):98-103.

22. Kim JY, Lee KT, Young KW, Son SW. The Comparasion of Ultrasonographic Size of Morton's Neeuroma measured to Actual Size. J Korean Foot Ankle Soc. 2006; 10(1):80-3.

23. Breusch SJ, Sharp RJ, Wenz W, Taylor LJ. Morton's neuroma following first metatarsal osteotomy. Orthopedics. 1998;21(12):1287-8.

24. Gudas CJ, Mattana GM. Retrospective analysis of intermetatarsal neuroma excision with preservation of the transverse metatarsal ligament. J Am Podiatr Med Assoc. 1986;76:459-63.

25. Addante JB, Peicott PS, Wong HY, Brooks DL. Interdigital neuromas: results of surgical excision of 152 neuromas. J Am Podiatr Med Assoc. 1986;76:493-5.

26. Esling F, Martin JM, Py E, Ngyen L, Savornin C. Le névrome de Morton: Résultats du traitement chirurgical par neurectomie, à propos de 36 cas revus. E-memoires de l'Academie Nationale de Chirurgie $2002 ; 1(1): 13-16$.

27. Fernández JM, Camacho J, Fernández LJ. Neuritis interdigital en el pie: Resultados en el Centro Médico ABC. An Med. 2002; 47:85-90, 2002.

28. Hewitt SM, Kilmartin TE, O'Kane C. A retrospective audit on the role of sonographical interpretation and localisation of intermetatarsal neuroma in the surgical management of Morton's neuroma. Brit J Podiatr. 2007; 10(3):99-103. 
29. Nery CA, Barroco RS, Furlan C, Tardini CH, Cemin FS, Mombach RG. Tratamento do neuroma de Morton via plantar: avaliação retrospectiva dos resultados cirúrgicos. Acta Ortop Bras. 2007; 15(1):55-8.

30. Lee SY, Jung Y, Lee S. Ultrasonography of Morton's Neuroma Accompanied with Interdigital Bursitis. J Korean Acad Rehabil Med. 2009;33(4):386-91.

31. Soo JM, Perera SD, Payne S. The use of ultrasound in diagnosing Morton's neuroma and histological correlation .Ultrasound 2010;18:14-7.

32. Faraj AA, Hosur A. The outcome after using two different approaches for excision of Morton's neuroma. Chin Med J (Engl) 2010;123:2195-8.

33. Valero J, Gallart J, Gonzalez D, Deus J, Lahoz M. Multiple Interdigital Neruromas. A Retrospective Study of 279 Feet With 462 Neuromas. J Foot Ankle Surg. Article in press. YJFASS1678_proof.26 June 2014.

34. Hassouna H, Singh D. Morton's metatarsalgia: Pathogenesis, aethiogy and current management. Acta Orthop Belg. 2005;71:646-55.

35. Franson J, Baravarian B. Intermetatarsal compression neuritis. Clin Podiatr Med Surg. 2006;23(3):569-78.

36. Wilson S, Kuwada G. retrospective study of the use of va plantar transverse incision versus dorsal incision for excision nof neuroma. J Foot Ankle Surg. 1995;34(6):537-40.

37. Akermark C1, Crone H, Skoog A, Weidenhielm L. A prospective randomized controlled trial of plantar versus dorsal incisions for operative treatment of primary Morton's neuroma. Foot Ankle Int. 2013;34(9):1198-204.

38. Biasca N, Zanetti M, Zollinger H.. Outcomes after partial neurectomy of Morton's neuroma related to preoperative case histories, clinical findings, and findings on magnetic resonance imaging scans. Foot Ankle Int. 1999;20(9):568-75.

39. Lee KT, Lee YK, Young KW, Kim HJ, Park SY. Results of operative treatment of double Morton's neuroma in the same foot. J Orthop Sci. 2009;14(5):574-8..

40. Kasparek M, Schneider W. Surgical treatment of Morton's neuroma: clinical results after open excision. Int Orthop. 2013;37(9):1857-61.

41. Diebold PF, Delagoutte JP. La neurolyse vraie dans le traitement du névrome de Morton. Acta Orthop Belg. 1989;55(3):467-71.

42. Nemoto K, Mikasa M, Tazaki K, Mori Y. Neurolysis as a surgical procedure for Morton's neuroma. Nihon Seikeigeka Gakkai Zasshi. 1989;63(5):470-4.

43. Okafor B, Shergill G, Angel J. Treatment of Morton's neuroma by neurolysis. Foot Ankle Int. 1997;18(5):284-7.

44. Bregman PJ. Radiofrequency thermoneurolysis for the treatment of Morton's neuroma. J Foot Ankle Surg. 2012;51(3):405; author reply 405. doi: 10.1053/j.jfas.2012.02.004.

45. Moore JL, Rosen R, Cohen J, Rosen B. Radiofrequency thermoneurolysis for the treatment of Morton's neuroma. J Foot Ankle Surg. 2012;51(1):20-2.

46. Argüelles R. Manual de Patología Quirúrgica (Tomo II). Barcelona-Madrid: Editorial Científico Médica, 1940; p.509.

47. Señarís J, Baamonde C, Aizpurúa J, Prego A, Arriaza R. Resultados del tratamiento quirúrgico de los neuromas interdigitales mediante técnicas mínimamente invasivas. Acta Orthop Gallega 2007;3(1):3-6.

48. Samrendu KS, James PI, Chistopher PC. The surgical treatment of Morton's neuroma. Current Orthopaedics 2005;19:379-84.

49. Kilmartin TE, Wallace WA. Effect of pronation and supination orthosis on Morton's neuroma and lower extremity function. Foot Ankle Int. 1994;15(5):256-62. 